\title{
Fully automated RF-thermal stress workbench with S-parameters tracking for GaN reliability analysis
}

\author{
D. Saugnon ${ }^{1,2}$, J.G. Tartarin ${ }^{1}$, B. Franc ${ }^{1}$, H. Maher ${ }^{2}$, F. Boone ${ }^{2}$ \\ ${ }^{1}$ LAAS-CNRS, University of Toulouse, CNRS, UPS, Toulouse, France \\ ${ }^{2}$ Laboratoire Nanotechnologies Nanosystèmes, CNRS UMI-3463, Institut Interdisciplinaire d'Innovation Technologique, \\ University of Sherbrooke, Sherbrooke, QC, Canada
}

tartarin@1aas.fr

\begin{abstract}
The rapid development of III-V technologies for telecommunication and radar markets need the meeting of performances (power, frequency) criteria as well as reliability assessment. Nitride HEMT technologies are known to reveal a large variety of failure electrical signatures, and it is also largely accepted that multi-tools (multi physics) approaches is the only suitable way to understand the failure mechanisms and to improve the technologies. Experimental stress workbenches usually allow to track a given number of static/dynamic parameters, but specific characterization are only performed at initial and final steps on the devices. This paper proposes a new approach with Sparameters measurement performed during RF stresses without removing the devices under test (in a thermally controlled oven). Then intermediate knowledge of the electrical (small signal) behavior of the devices can be assessed, and crossed with largesignal and static time-dependent signatures.
\end{abstract}

Keywords - Gallium nitride, HEMTs, Microwave transistors, C-band, Semiconductor device reliability, Life testing.

\section{INTRODUCTION}

III-V wide bandgap disruptive technologies take advantage over conventional GaAs and $\mathrm{Si}(\mathrm{Ge})$ technologies for high power and high frequency wireless applications such as base stations and backhaul infrastructure [1], [2], defense and military applications (radar, jamming, counter-measures, guided weapons, etc.) [3], [4], broadcast and communication satellites (SatCom) [5]-[8]. High electron mobility transistor (HEMT) is now reaching a Technology Readiness Level (TRL) sufficiently high to address those markets, but a margin still exists to fully exploit the Nitride technologies capacities. The main improvement area concerns the reliability enhancement of gallium nitride ( $\mathrm{GaN}$ ) HEMT devices, i.e. the ability of those technologies to sustain high power at elevated junction temperatures over a long period. Then, stresses under realistic conditions are needed to assess the stability of the DC and radio frequency $(\mathrm{RF})$ electrical parameters. However, before a final qualification, many steps must be overcome to improve the technological process [9]. In this paper, a new thermal and RF stress setup is proposed to allow intermediate dynamic characterizations during a stress campaign, without removing the devices from test bench (i.e. mastering the delays before/after the application of the stress and ensuring the connectivity stability over the total time of the stress campaign). In the first section of this paper, a synopsis of the workbench is proposed, as well as the associated calibration tests. The devices under test (DUTs) and RF stress conditions are proposed in the second section, and the results are mentioned in the next section. Finally, conclusions are drawn concerning the pertinence of such necessity to merge extra-characterization tools with conventional RF stress benches, by providing more data for the final analysis and for reducing the risk of speculative outcomes.

\section{RF AND THERMAL STRESS WORKBENCH WITH S- PARAMETERS MEASUREMENT FACILITIES}

Fig. 1 shows the synopsis of the experimental stress bench. The bench allows to track the conventional DC and RF markers (drain-source currents $\mathrm{I}_{\mathrm{DS}}$, output powers $\mathrm{P}_{\mathrm{OUT}}$ ) and temperature inside the Espec SU-241 thermal chamber. A set of SPDT and SP4T switches enable S-parameters measurement capabilities (Agilent N5230C VNA) outside the main stress measurement chain. It is a major issue during RF stresses to keep constant or to get feedback over stability of all the circuitry of the stress workbench, out of the DUTs. For that purpose, temperature of the oven and that of the ambient room are also monitored $\left( \pm 0.1^{\circ} \mathrm{C}\right.$ measurement accuracy). More critical, as four different channels are available in our configuration, three channels are devoted to DUTs, and a control channel is saved to secure the analysis with potential drifts from the driver module (synthesizer and power amplifier).

This experimental setup allows different temperature cycling profiles between $-40 \quad{ }^{\circ} \mathrm{C}$ and $+150{ }^{\circ} \mathrm{C}$

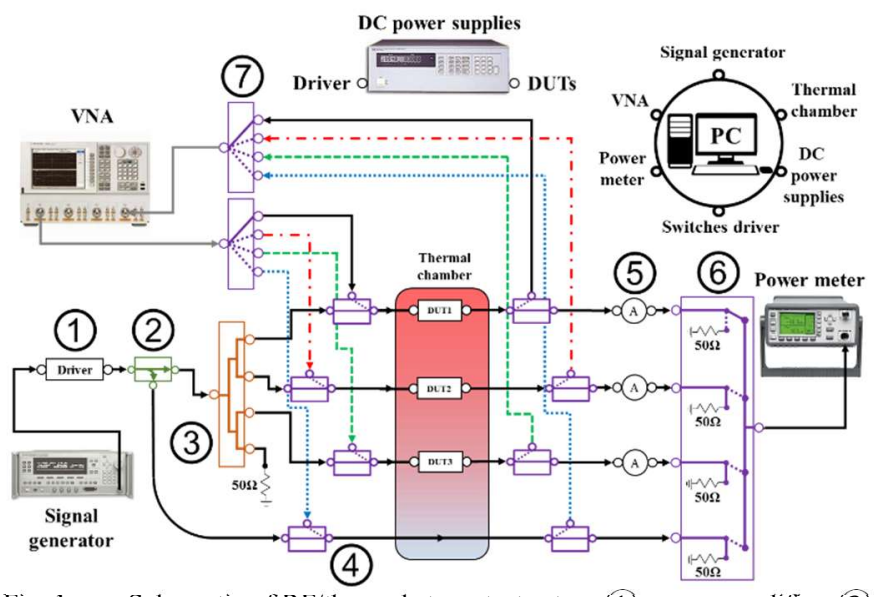

Hig. 1. Schematic of $\mathrm{RH} /$ thermal stress test setup: (1) power amplifier, (2) coupler, (3) four-way power divider, (4) SPDT, (5) attenuators, (6) SP4T 50 $\Omega$ RF load terminations (7) SP4T. 
(triangular/rectangular or constant temperature profiles). For the convenience of the targeted goals, only constant ambient temperature profile $\left(+25{ }^{\circ} \mathrm{C}\right)$ is shown in this paper, to understand the mechanisms undergoing the reliability (electrical behavior: partition between RF dynamical stress and low-level signal signature, recoverable or permanent degradation effects) of the devices at a given ambient temperature from those activated at low/high temperatures during cycling.

Preliminary calibration is performed to assess the stability of S-parameters (each path) and the eventual compensation of the drift on the RF stress paths (post compensation) on each of the four channels. In VNA mode, each of the four paths is calibrated independently. The stability of the calibration has been verified at ambient temperature over 500 hours, and for $-40{ }^{\circ} \mathrm{C} /+100{ }^{\circ} \mathrm{C}$ thermal cycling over one week. Less than $\pm 0.05 \mathrm{~dB}\left( \pm 1^{\circ}\right)$ drift at ambient and $\pm 0.04 \mathrm{~dB}\left(+2^{\circ}\right)$ drifts for $-40{ }^{\circ} \mathrm{C} /+100{ }^{\circ} \mathrm{C}$ thermal profiles. The total channel $\mathrm{RF}$ power fluctuations correspond to the drift of the power source HP $83650 \mathrm{~B}$ (10 MHz to $50 \mathrm{GHz}$ ), coupled with the HP 8487A power sensor $(50 \mathrm{MHz}$ to $50 \mathrm{GHz}$ ) and to the Agilent E4418B power meter measurement uncertainty. The induced temperature total power drift at $-40{ }^{\circ} \mathrm{C},+25^{\circ} \mathrm{C}$ and $+100{ }^{\circ} \mathrm{C}$ is respectively of $\Delta \mathrm{P}_{@-40^{\circ} \mathrm{C}} \leq+0.01 \mathrm{~dB}, \Delta \mathrm{P}_{@+25^{\circ} \mathrm{C}} \leq+0.03 \mathrm{~dB}$ and $\Delta \mathrm{P}_{@+100{ }^{\circ} \mathrm{C}} \leq \pm 0.02 \mathrm{~dB}$.

$\mathrm{P}_{\mathrm{OUT}}$ and $\mathrm{I}_{\mathrm{DS}}$ (drain-source current quiescent bias condition under RF input signal) are saved each 10 minutes. S-parameters acquisition is performed at $\mathrm{T}_{0}$, after 10 minutes of stress and then each 6 hours of stress period. Acquisitions times can be selected at the user convenience for a given stress campaign.

RF paths at room temperature out of the stress chamber leads to small thermal drifts (in spite of only $\pm 1{ }^{\circ} \mathrm{C}$ daily drift); the impact on the power amplifier driver stability is compensated in real time using the reference path measurement and the temperature profile.

\section{RF STRESSES AT DIFFERENT COMPRESSION POINTS}

Devices under test are developed using $\mathrm{GaN}$ on $\mathrm{SiC}$ substrate process with $24 \% \mathrm{Al}$ content. HEMT devices feature $2 \times 75 \mu \mathrm{m}^{2}$ gate width and $0.25 \mu \mathrm{m}$ gate length $\left(\mathrm{F}_{\mathrm{t}}=40 \mathrm{GHz}\right.$ and $\mathrm{F}_{\max }=100 \mathrm{GHz}$ ) [10], and are packaged on an RF test fixture for easier connectivity in the thermal chamber. Devices are biased under A-class operation. To prove the pertinence of the proposed approach, only one device from the batch under test is discussed in this paper (\#TR2). Moreover, in a first approach, only ambient stresses are driven at $1 \mathrm{~dB}, 3 \mathrm{~dB}$ and $5 \mathrm{~dB}$ output compression points, using a $4.2 \mathrm{GHz}$ continuous wave signal carrier. This thermally stable condition allows us to focus specific mechanisms without considering changes in the temperature. In Fig. 2 and Fig. 3 are respectively presented the $\mathrm{DC}$ and power evolution plots versus time for $1 \mathrm{~dB}, 3 \mathrm{~dB}$ and two characterizations at $5 \mathrm{~dB}$ (namely \#A and \#B).

Between each RF-stress, a positive DC voltage $\mathrm{V}_{\mathrm{GS}}=1 \mathrm{~V}$ (shorted drain) is applied on the DUTs to recover initial electrical state for each device (due to possible accumulation of charges during the stress): some recoverable effects due to the presence of mobile charges (and also possibly to fixed charges

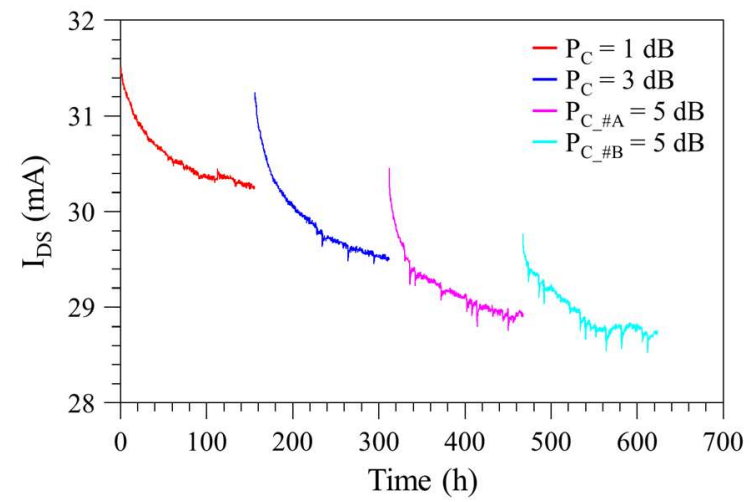

Fig. 2. Experimental RF stress results for \#TR2 at chamber temperature of $+25^{\circ} \mathrm{C}$. $\mathrm{I}_{\mathrm{DS}}$ vs time at $1 \mathrm{~dB}, 3 \mathrm{~dB}$ and $5 \mathrm{~dB}$ (\#A and \#B) compression points for quiescent point at $\mathrm{V}_{\mathrm{DS}}=15 \mathrm{~V}$ and $\mathrm{I}_{\mathrm{DS}}=35 \mathrm{~mA}$.

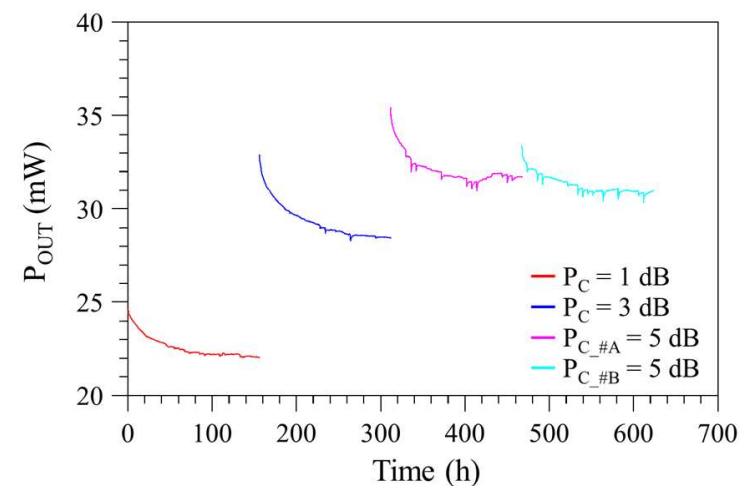

Fig. 3. Experimental RF stress results for \#TR2 at chamber temperature of $+25^{\circ} \mathrm{C}$. P Out vs time at $1 \mathrm{~dB}, 3 \mathrm{~dB}$ and $5 \mathrm{~dB}$ (\#A and \#B) compression points for quiescent point at $\mathrm{V}_{\mathrm{DS}}=15 \mathrm{~V}$ and $\mathrm{I}_{\mathrm{DS}}=35 \mathrm{~mA}$.

if not bind to a deep center) under the gate can affect initial state of the devices before each stress campaign. After each RF stress period ( $1 \mathrm{~dB}, 3 \mathrm{~dB}, 5 \mathrm{~dB}$ \#A and \#B compression point), the drops of drain-source current are $3.9 \%, 5.6 \%, 5 \%$ and $3.4 \%$ respectively. The drops for output power are $10.4 \%, 13.5 \%$, $10.5 \%$ and $7.2 \%$ respectively. It is obvious that the change in the static and dynamic plots are more pronounced at elevated compression levels certainly due to higher non-linear effects, as will be discussed in paragraph IV. It can also be noticed that the drop slopes in the first 20 hours are more severe when applying higher RF stress levels; and then after 50 hours, an asymptotic trend can be merged between $1 \mathrm{~dB}, 3 \mathrm{~dB}$ and $5 \mathrm{~dB}$ stress conditions. Moreover in Fig. 3, monotonous decreasing trends are visible on $\mathrm{P}_{\mathrm{OUT}}$ for $1 \mathrm{~dB}$ and $3 \mathrm{~dB}$ compression levels, whereas more dispersive effects are plotted over time for \#A $5 \mathrm{~dB}$ plot. Then a second \#B $5 \mathrm{~dB}$ stress is performed, to eventually discriminate between permanent or recoverable degradation mechanisms, or to evidence at least the activation of a different degradation signature.

\section{DISCUSSIONS ON DC/RF STRESSES AND S-PARAMETERS MEASUREMENTS}

The correlation between static current drops and RF power variation is a well-known signature of GaN technologies [11]; this states that the RF variation at the output of the devices is 


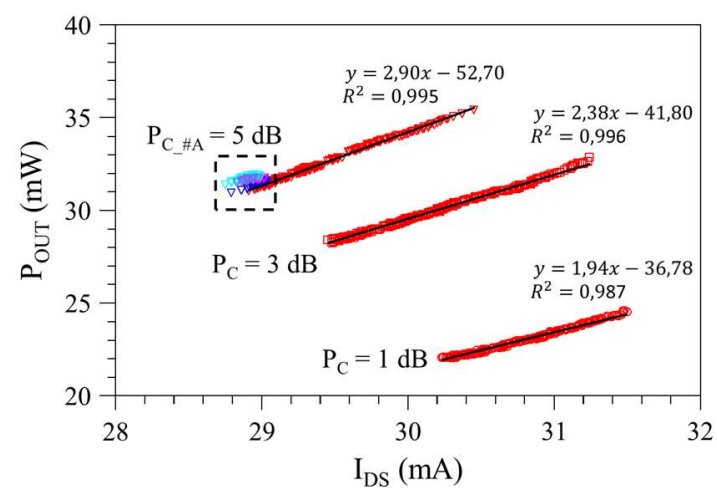

Fig. 4. Correlation between $P_{\text {OUT }}$ and $I_{D S}$ for device \#TR2 at $1 \mathrm{~dB}, 3 \mathrm{~dB}$ and $5 \mathrm{~dB}(\# \mathrm{~A})$ compression point.

not related to a degradation of the 2DEG channel, but more likely to a change of the gated zone (under or below the 2DEG) [12]. Fig. 4 shows the correlation of the degradation rates between $P_{\text {OUT }}$ and $\mathrm{I}_{\mathrm{DS}}$ at $1 \mathrm{~dB}, 3 \mathrm{~dB}$ and $5 \mathrm{~dB}$ \#A compression levels (\#B not shown here for a greater clarity). From linear trends given by plots in Fig. 4 as $P_{O U T}=a_{x d B} \cdot I_{D S}+b_{x d B}$ for each " $\mathrm{x} \mathrm{dB}$ " compression point, it can be noticed the increase of the slope ' $a$ ' with the applied RF power. This trend evidences the larger effect of higher RF swings on Pout drifts, as a marker of the 2DEG control degradation.

As noticed in Fig. 3 on the non-monotonic behavior of Pout during the RF stress at $5 \mathrm{~dB}$ \#A compression point, Fig. 4 still exhibits a deviation from the main linear trend (red plot) at lower output powers (specified by a rectangular zone, and corresponding to the higher time sequence of stress). Pout and $\mathrm{I}_{\mathrm{DS}}$ relationships cannot be accounted by a single linear function. Fig. 5a focuses on this rectangular plot from Fig. 4, where more than four degradation rates are evidenced over the stress duration at $5 \mathrm{~dB}$ \#A (next noticed as (1)(2)(4)(5), as (3) only concerns a transition phase); arrows are used to evidence the time sequences from phase (1) to phase (5). The first trend noticed as phase (1) relates to the main behavior already discussed in Fig. 4 (red plots). Then a recovery phase (2) still featuring the same signature than during phase (1) can also be noticed with an increase in Pout versus time in Fig. 5b. Then after a transition phase (3) (with no associated linear trend), the device switches to another drop phase (4), and a new recovery phase 5 (also visible in Fig. 5b); however these two last phases (4) (5) feature different linear coefficients ' $a$ ' and ' $b$ ' than during phases (1)(2). This new signature should be the manifestation of a new degradation law; to evidence this new signature, a second \#B stress is performed at $5 \mathrm{~dB}$ after a recovery period and after the application of a positive $\mathrm{DC}$ gate voltage. Results are given in Fig. 2 and Fig. 3 for \#B stress. During \#B stress period, only two phases are evidenced, next noticed as $\# \mathrm{~B}$ (1) and $\# \mathrm{~B}(2)$.

Fig. 6 plots the linear ' $a$ ' and ' $b$ ' coefficient for each $1 \mathrm{~dB}$, $3 \mathrm{~dB}$ and \#A, \#B $5 \mathrm{~dB}$ stresses campaigns on \#TR2 device. First it can be noticed the linear regression of ' $a$ ' and ' $b$ ' coefficients with the RF compression level, evidencing the impact of the non-linear effects on the degradation law. A first mechanism gathers the $1 \mathrm{~dB}, 3 \mathrm{~dB}$ and $5 \mathrm{~dB} \# \mathrm{~A}(1),(2)$ and $\# \mathrm{~B}(1)$ under a linear regression of the ' $a$ ' and ' $b$ ' coefficients, revealing the

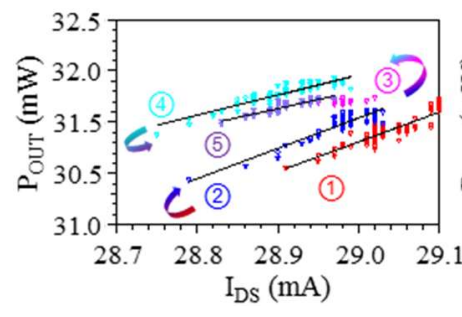

(a)

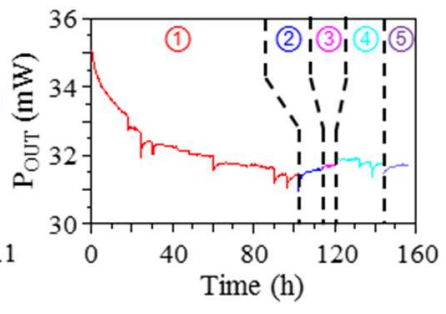

(b)
Fig. 5. Correlation between $\mathrm{P}_{\mathrm{OUT}}$ and $\mathrm{I}_{\mathrm{DS}}$ for device \#TR2 at $5 \mathrm{~dB}$ output compression. (a) focuses on the rectangular dotted zone of Fig. 4. Arrows in (a) indicate the time sequence, segregating different zones-mechanisms noticed from 1 to 5 as also specified in (b).

power-law dependence of these parameters according to the compression level of the device. Then a second mechanism (only related to $5 \mathrm{~dB}$ stresses) featuring lower ' $a$ ' and ' $b$ ' coefficients is also plotted in Fig. 6, revealing the activation of a new $\mathrm{P}_{\text {OUT }}-\mathrm{I}_{D S}$ dependence law (related to \#A(4), (5) and \#B(2) phases). It is also convenient to notice that the second $\# \mathrm{~B} 5 \mathrm{~dB}$ stress features a degradation law according to the first mechanism, and then switches to the second mechanism after 18 hours. This behavior should rise from the degradation of the control input capacitance $\mathrm{C}_{\mathrm{GS}}$ during stress (thus affecting the controlled current source of the 2DEG), or also from the activation of charges (fixed or mobiles) still located under the gated zone (thus affecting the internal voltage of the controlled current source of the 2DEG).

Thus, according to the time dependent mechanisms evidenced from Fig. 1 to Fig. 6, it is not possible to conclude yet about the stability of the electrical model of the device under test; the knowledge of S-parameters are used to bring additional data concerning the gain $\mathrm{S}_{21}$ related to the carriers in the 2DEG, the reflection coefficient $S_{11}$ revealing a possible change in $\mathrm{C}_{\mathrm{GS}}$ command capacitance (space charge region degradation), and eventually $S_{12}$ isolation (still related to space charge region between gate and drain $\mathrm{C}_{\mathrm{GD}}$ ). Using the first order equation of the dynamic drain current $I_{D S}=g_{m} . V_{G S_{\text {_int }}}$, where $\mathrm{g}_{\mathrm{m}}$ is the transconductance gain (i.e. $\approx \mathrm{S}_{21}$ ) and $\mathrm{V}_{\mathrm{GS}}$ int is the intrinsic command voltage at the $\mathrm{C}_{\mathrm{GS}}$ terminals, S-parameters can bring useful information to discriminate between the proposed hypothesis.

In Fig. $7 \mathrm{a}$, the linear gain parameter $\mathrm{S}_{21}$ is plotted versus frequency at different stress times (here for the $5 \mathrm{~dB}$ \#A stress). The previously evidenced changes in $P_{O U T}$ and $I_{D S}$ are again demonstrated in the drift of the dynamic gain of the device.

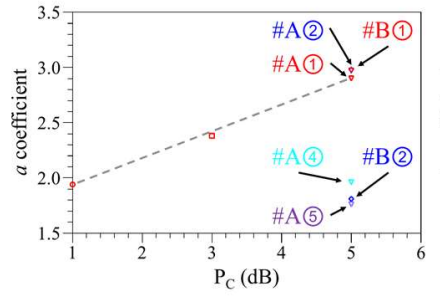

(a)

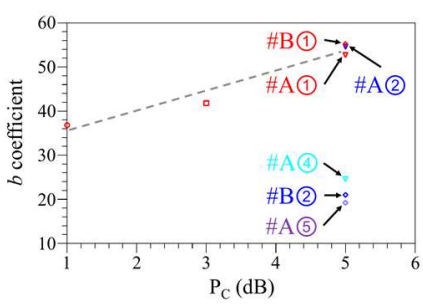

(b)
Fig. 6. Affine function coefficients for device \#TR 2 at $1 \mathrm{~dB}, 3 \mathrm{~dB}$ and $5 \mathrm{~dB}$ (\#A and \#B) output compression. (a) and (b) shows the relation between the activated degradation mechanism number during the stress. 


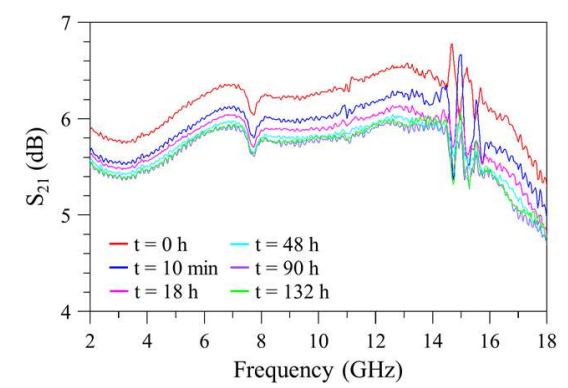

(a)

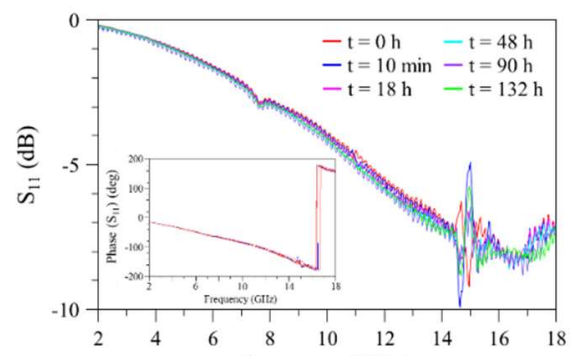

(b)

Fig. 7. S-parameters evolution for $\mathrm{P}_{\mathrm{C}_{-} \# \mathrm{~A}}=5 \mathrm{~dB}$.

According to the previous expression of the dynamic $\mathrm{I}_{\mathrm{DS}}$ current, then $\mathrm{S}_{11}$ (i.e. $\approx \mathrm{C}_{\mathrm{GS}}$ ) is also plotted in Fig. $7 \mathrm{~b}$; the magnitude/phase stability evidences the constant $\mathrm{C}_{\mathrm{GS}}$ capacitance value during the stress. Then, the variations discussed in Fig. 1 to Fig. 6 are not associated with a structural degradation of the small-signal model of the HEMT under test. The drift in $\mathrm{I}_{\mathrm{DS}}$ is then attributed to a variation of $\mathrm{V}_{\mathrm{GS}}$ int due to the activation of charges under the gate. These charges act like a serial biasing voltage source that makes $\mathrm{V}_{\mathrm{GS}}$ int $($ time) fluctuate; it can be expressed as $V_{G S \_ \text {int }}($ time $)=V_{G S \_ \text {ext }}$. $V_{\text {charg }}$ (time) where $\mathrm{V}_{\mathrm{GS} \text { ext }}$ is the external biasing voltage and $\mathrm{V}_{\text {charges }}($ time) is the time dependent generator bind to charges fluctuations [13] under elevated stress conditions. It would be speculative to conclude about the nature of the charges under concern; however, thanks to S-parameters analysis, it can be concluded about the integrity of the input (output) terminals of the DUT. Then, the decreasing (resp. increasing) time trends associated to the first signature revealed at $1 \mathrm{~dB}, 3 \mathrm{~dB}, 5 \mathrm{~dB} \# \mathrm{~A}(1)$ and $\# \mathrm{~B}$ (1) (resp. $5 \mathrm{~dB} \# \mathrm{~A}$ (2)) is associated to one or more type of charges moving the $\mathrm{V}_{\mathrm{GS} \text { int }}($ time) control voltage toward negative (resp. positive) values. The same conclusion can be developed concerning the second signature only revealed at $5 \mathrm{~dB} \# \mathrm{~A}(4)$, (5) and \#B(2), probably involving charges in a different zone under the gate.

\section{CONCLUSION}

This paper presents a stress analysis on GaN device(s) using a fully embedded RF-thermal stress workbench featuring Sparameters intermediate measurement facilities. From the usually discussed RF and DC evolutions with time (and stress type) found in the literature, additional small-signal data are available to conclude about the stability (or change) in specific parts of the electrical model of the DUT. Then correlation between time evolving signatures under the application of a stress, and other analysis tools (here S-parameters) enhance the data collection to reinforce the analysis concerning the undergoing mechanisms. In this paper, the increase of the stress power reveals different linear trends associated to two different signatures of degradation; from S-parameters analysis, it is concluded about the time-fluctuation of charges under the gated zone of the device under test. Moreover, the proposed automated stress workbench can be easily embedded with different new equipment such as large-signal VNA, or also ( $\mathrm{LF} / \mathrm{HF}$ ) noise figure analyzers to provide the users with more pertinent data collected during the stress session.

\section{ACKNOWLEDGMENT}

We acknowledge support from GANEX (ANR-11-LABX0014). GANEX belongs to the public funded 'Investissements d'Avenir' program managed by the French ANR agency.

\section{REFERENCES}

[1] A. S. M. Alqadami, S. Madhuwantha, R. Farrell, and J. Dooley, "A 5 W High Efficiency Class AB Power Amplifier for LTE Base Station Application," in 28th Irish Signals and Systems Conference (ISSC), 2017, pp. 1-5.

[2] V. Camarchia, R. Quaglia, C. Ramella, and M. Pirola, "Power amplifier MMICs for $15 \mathrm{GHz}$ microwave links in $0.25 \mu \mathrm{m}$ GaN technology," in Integrated Nonlinear Microwave and Millimetre-wave Circuits Workshop (INMMiC), 2017, pp. 1-3.

[3] H. Q. Tao, W. Hong, B. Zhang, and X. M. Yu, "A Compact 60W X-Band GaN HEMT Power Amplifier MMIC," IEEE Microw. Wirel. Components Lett., vol. 27, no. 1, pp. 73-75, 2017.

[4] M. Rudolph, "GaN HEMTs for low-noise amplification — status and challenges," in IEEE Integrated Nonlinear Microwave and Millimetrewave Circuits Workshop (INMMiC), 2017, pp. 1-4.

[5] P. Waltereit, J. Kühn, R. Quay, F. Van Raay, M. Dammann, M. Cäsar, S. Müller, M. Mikulla, O. Ambacher, J. Lätti, M. Rostewitz, K. Hirche, and J. Däubler, "High efficiency X-band AlGaN/GaN MMICs for space applications with lifetimes above $10^{\wedge} 5$ hours," in IEEE European Microwave Integrated Circuit Conference (EuMIC), 2012, pp. 123-126.

[6] A. Katz, J. Macdonald, R. Dorval, B. Eggleston, P. Drexler, and C. Liang, "High-Efficiency High-Power Linearized L-Band SSPA for Navigational Satellites," in IEEE MTT-S International Microwave Symposium (IMS), 2017, pp. 1834-1837.

[7] M. van Heijningen, P. de Hek, C. Dourlens, P. Fellon, G. Adamiuk, N. Ayllon, and F. van Vliet, "C-Band Single-Chip Radar Front-End in AlGaN/ GaN Technology," IEEE Trans. Microw. Theory Tech., vol. 65, no. 11 , pp. $4428-4437,2017$.

[8] D. H. Shin, I. B. Yom, and D. W. Kim, "X-Band GaN MMIC Power Amplifier for the SSPA of a SAR System," in IEEE International Symposium on Radio-Frequency Integration Technology (RFIT), 2017, pp. 93-95.

[9] K. Hirche, J. Lätti, M. Rostewitz, K. Riepe, B. Lambert, R. Lossy, J. Würfl, P. Waltereit, J. Kühn, R. Quay, F. Van Raay, M. Dammann, M. Casar, S. Muller, D. Marcon, S. Decoutere, M. Auf der Maur, A. Di Carlo, J. Pomeroy, and M. Kuball, "GaN Reliability Enhancement and Technology Transfer Initiative $\left(\right.$ GREAT $\left.^{2}\right)$, , in Abstract of ESA/ESTEC Contract No. 21.499/08/NL/PA, pp. 1-8.

[10] G. Soubercaze-Pun, J. G. Tartarin, L. Bary, J. Rayssac, E. Morvan, B. Grimbert, S. L. Delage, J.-C. De Jaeger, and J. Graffeuil, "Design of a $\mathrm{X}$-band GaN oscillator: from the low frequency noise device characterization and large signal modeling to circuit design," in IEEE MTT-S International Microwave Symposium (IMS), 2006, pp. 747-750.

[11] J. Joh, J. A. del Alamo, U. Chowdhury, and J. L. Jimenez, "Correlation between RF and DC reliability in GaN high electron mobility transistors," Reliab. Compd. Semicond. Work., pp. 185-194, 2008.

[12] J. G. Tartarin, "Diagnostic Tools For Accurate Reliability Investigations of GaN Devices," 21st Int. Conf. Noise Fluctuations, pp. 452-457, 2011.

[13] O. Lazar, J. G. Tartarin, B. Lambert, C. Moreau, and J. L. Roux, "Correlation between transient evolutions of the gate and drain currents in AlGaN/GaN technologies," Microelectron. Reliab., vol. 55, no. 9, pp. 1714-1718, 2015. 